\title{
Microfluidic Synthesis of Cell-Type-Specific Artificial Extracellular Matrix Hydrogels
}

\author{
Simone Allazetta, Tanja C. Hausherr, ${ }^{\dagger}$ and Matthias P. Lutolf* \\ Laboratory of Stem Cell Bioengineering, Institute of Bioengineering, Ecole Polytechnique Fédérale de Lausanne (EPFL), Lausanne, \\ Switzerland
}

\section{Supporting Information}

ABSTRACT: Droplet microfluidic technology is applied for the high-throughput synthesis via Michael-type addition of reactive, micrometer-sized poly(ethylene glycol) (PEG) hydrogels ("microgels") with precisely controlled dimension and physicochemical properties. A versatile chemical scheme is used to modify the reactive PEG microgels with tethered biomolecules to tune their bioactive properties for the bioreactor culture and manipulation of various (stem) cell types.

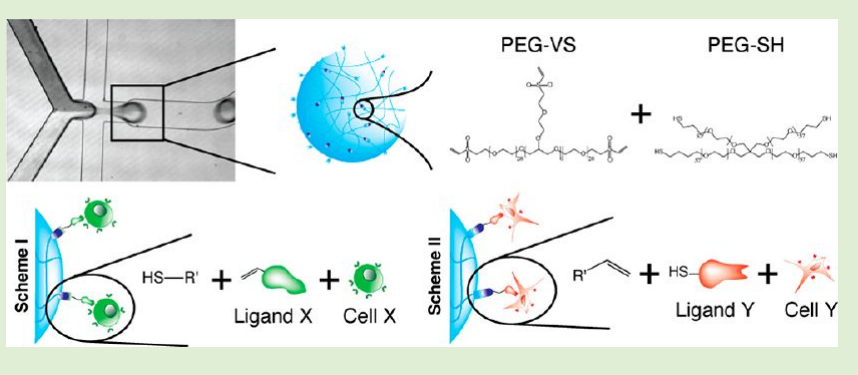

aECM gels for applications in stem cell biology and biotechnology.

As a first target to apply such a platform technology, we focused on the generation of aECM microgels for the adherent culture of stem cells in bioreactors, a powerful method to generate large amounts of cells for clinical purposes. ${ }^{12,13}$ Accordingly, such microcarriers fabricated from different materials including polystyrene, cellulose, glass, or dextran have been used for several decades in the pharmaceutical industry, for example, for the large-scale production of proteins or viruses. ${ }^{14,15}$ However, despite the fact that several microcarrier families have been tested for the expansion of stem cells in bioreactors, ${ }^{16-24}$ to the best of our knowledge, none of the existing microcarrier systems can be readily engineered to confer biochemical and biophysical properties that are reminiscent of those of native stem cell niches.

Here we combine hydrogel engineering with droplet microfluidic technology ${ }^{25-27}$ to form aECM microgels in high-throughput. Michael-type addition chemistry was used to produce PEG microgels with modular dimension, stiffness and surface-tethered biomolecule composition. Such modularity allows tailoring microgel properties to a specific cell type and cell function of interest, for example to stimulate the robust expansion or differentiation of stem cells via display of specific niche components on the microcarrier surface.

\section{EXPERIMENTAL SECTION}

Materials. Poly(dimethylsiloxane) (PDMS) microfluidic chips were produced by using Sylgard 84 (Dow Corning). Four-arm PEG end-functionalized with thiols (4-arm-PEG-SH, $10 \mathrm{kDa}$ ) were obtained from NOF (Japan) and dissolved in bidistilled water. Vinylsulfone-functionalized 8-arm-PEG (8-arm-PEG-VS, $10 \mathrm{kDa}$ ) was

Received: January 4, 2013

Revised: February 21, 2013 


\section{A}

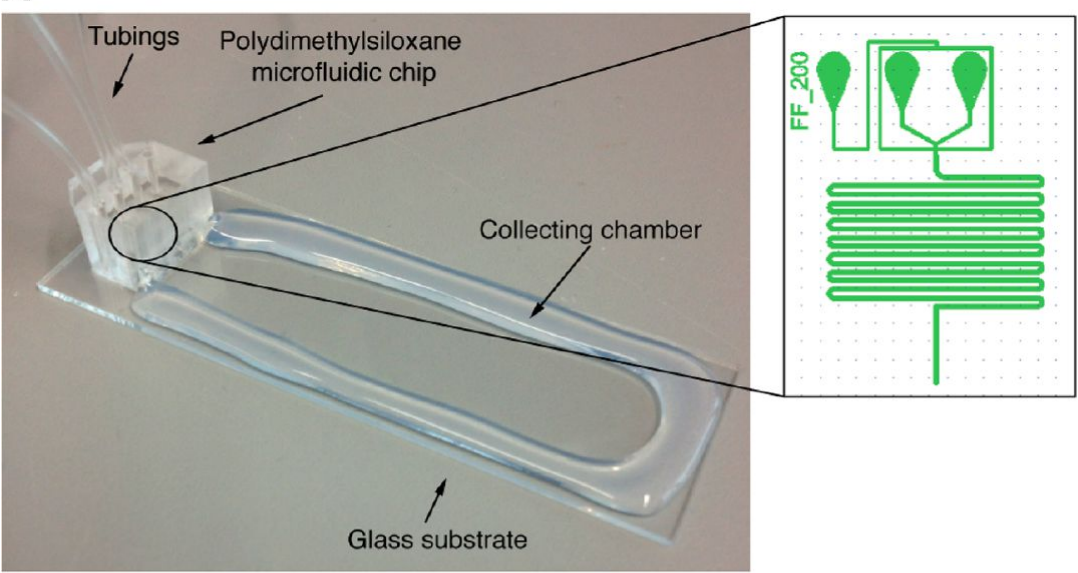

B

Flow focusing

C
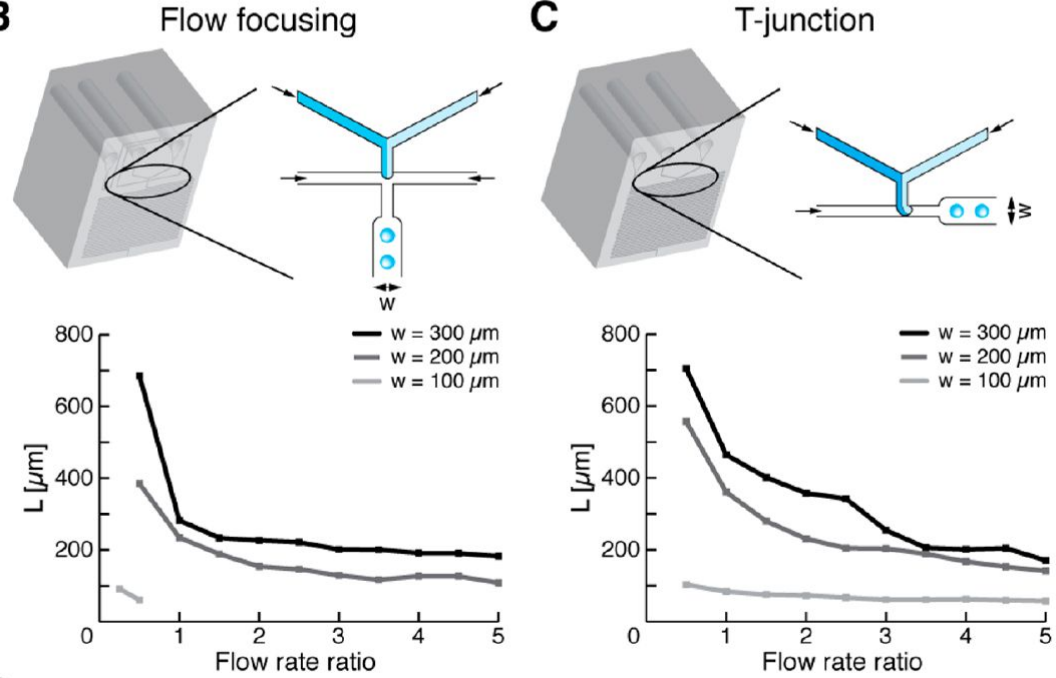

D

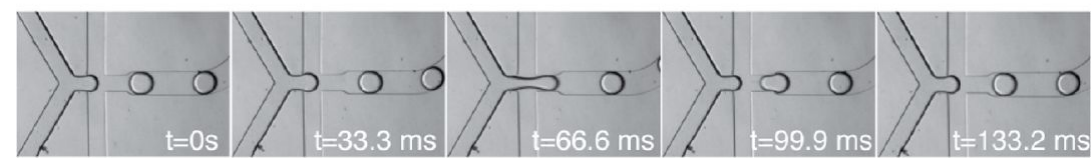

Figure 1. Characterization of microfluidic droplet formation. (A) Experimental setup used for microgel generation. A PDMS chip was bonded on top of a glass slide and a chamber was devised to collect microbeads. The enlargement shows the microfluidic layout for the $200 \mu \mathrm{m}$ flow-focusing design. (B, C) Flow focusing and T-junction designs used to generate microdroplets. Droplet length, $L$, was measured at different flow rate ratios and for different microchannels widths, $w$. Flow instabilities occurred in the $100 \mu \mathrm{m}$ flow focusing design and no droplets were generated for flow rate ratio higher than 0.5 . The light and dark blue colors indicate the presence of two different phases that do not mix each other under laminar flow conditions until the water stream is broken off by the organic phase. (D) Generation of a single droplet via flow focusing (200 $\mu \mathrm{m}$ channel width). The immiscible fluids form an interface at the junction; a droplet starts growing when the water stream penetrates into the main channel; the symmetric pressure gradient generated by the oil phase causes the breaking off of the droplet. ${ }^{41}$ Frequency of droplet formation $7.5 \mathrm{~Hz}$ (here).

synthesized as described ${ }^{28}$ and dissolved in triethanolamine $(0.3 \mathrm{M}$, $\mathrm{pH}$ 8). Amine-functionalized magnetic particles (size: $200 \mathrm{~nm}$ ) were supplied from Chemicell. Hexadecane and ABIL EM 90 were purchased from Sigma (U.S.A.) and Evonik Industries (Germany), respectively. Thiolated gelatin (GelinS) was purchased from Glycosan Biosystems (U.S.A.), and C-RGD (Ac-GRCGRGDSPG-NH ${ }_{2}$ ) was obtained from GL Biochem (China). Fibronectin fragment FN III ${ }_{9-10}$ bearing a free $\mathrm{N}$-terminal cysteine was a generous gift from Drs. Hubbell and Martino. ${ }^{29}$ Maleimide-activated NeutrAvidin and recombinant ProteinA were purchased from Pierce (Switzerland) and Biovision (Switzerland), respectively. Maleimide-PEG-NHS linker (M.W. $2000 \mathrm{Da}$ ) was obtained from JenKem Technology (U.S.A.). Biotinylation was performed using EZ-link maleimide-PEG -biotin $^{-}$ from Pierce (Switzerland). Human immunoglobulin ( $\mathrm{ggG}$ ) and bovine serum albumin (BSA) were purchased from Sigma (U.S.A.). AlexaFluor594 and AlexaFluor488 carboxylic acid, succinimidyl ester and AlexaFluor546 $\mathrm{C}_{5}$ maleimide fluorophores were obtained from Life Technologies (U.S.A.). Tris(2-carboxyethyl)phosphine hydrochloride (TCEP) and ethyl iodoacetate were obtained from Thermo Scientific (U.S.A.) and Sigma (U.S.A.), respectively.

Fabrication of Microfluidic Chips. Clewin software was used to draw microfluidic networks for droplet generation using a $\mathrm{T}$-junction and a flow focusing design composed of two microchannels for the water phase, intersected at $90^{\circ}$ angle with the main channel for the oil phase. PDMS chips were fabricated using conventional soft lithographic techniques. The $100 \mu \mathrm{m}$ deep channels with three different channel widths $(100,200$, and $300 \mu \mathrm{m})$ were patterned on SU8 masters. Microfluidic chips were bonded on oxygen plasma-activated glass slides (Figure 1A).

Microgel Generation. Computer-controlled syringe pumps (neMESYS from Cetoni, Germany) were used to control flow rates. Syringes were filled with PEG solutions and hexadecane with 2\% (w/ 


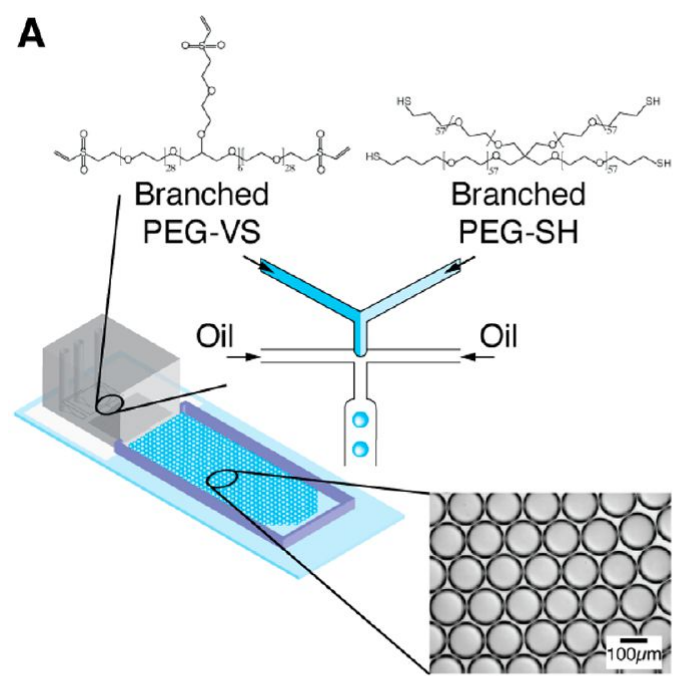

B

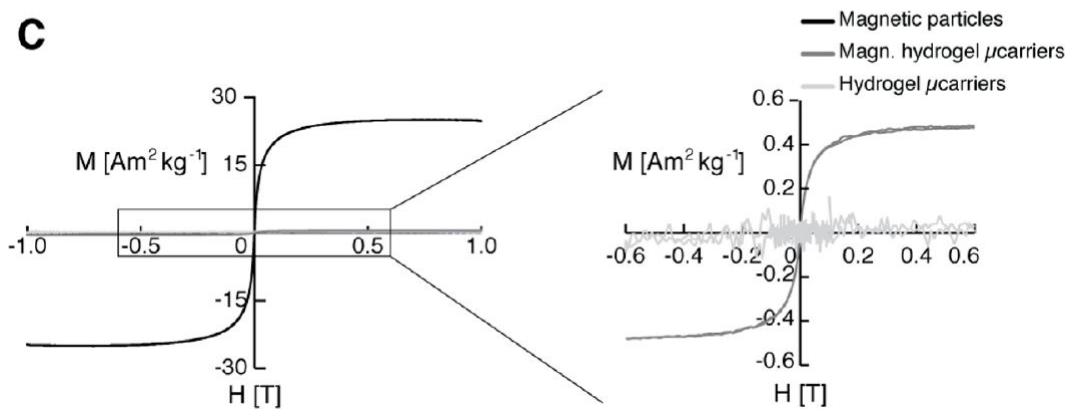

Figure 2. Microfluidic synthesis of PEG hydrogel microbeads. (A) Microgels are generated from reactive PEG macromers using a flow focusing microfluidic chip. A chamber collects microgels until completion of cross-linking. (B) Transparent magnetic microgels after removal of the oil phase. Histogram in lower panel shows the size distribution of magnetic microgels. (C) Hysteresis curves $(=$ applied magnetic field $(H)$ vs the magnetization $(M)$ ) demonstrating microgel magnetization. Magnetic particles and microgels with and without magnetic particles were analyzed. Magnified area shows magnetization of microgels compared to negative control.

v) ABIL EM surfactant used as oil phase. Tygon tubings were used to connect the syringes to the microfluidic chip inlets. Microgels were generated by loading two microfluidic channels with PEG precursors with concentrations depending on a specified molar excess of functional groups: for a $20 \%$ VS-excess, the concentrations of PEGVS and PEG-SH were $12 \%(\mathrm{w} / \mathrm{v})$ and $20 \%(\mathrm{w} / \mathrm{v})$, respectively. For a $20 \% \mathrm{SH}$-excess we chose $8.33 \%(\mathrm{w} / \mathrm{v})$ and $20 \%(\mathrm{w} / \mathrm{v})$ precursors, respectively. The oil phase was removed by filtering microgels with 70 $\mu \mathrm{m}$ cell strainers (BD Biosciences, U.S.A.) and extensive washing with PBS. Microgels were then swollen in PBS overnight. To render microgels paramagnetic, magnetic nanoparticles were mixed into the PEG-SH solution to a final concentration of $2.7 \mathrm{mg} / \mathrm{mL}$.

Measurements of Paramagnetism. Microgels with and without trapped magnetic nanoparticles were lyophilized and fixed with silicon grease (Dow Corning, Switzerland) into gelatin caps. A MicroMag 3900 Vibrating Sample Magnetometer (Laboratory for Micro- and Nanotechnology, ETH, Zurich) was used to assess magnetic properties.

Swelling Measurements. Swelling ratios $\left(Q^{\prime}\right)$ of microgel formulations were calculated as the ratio between bead volume in the oil phase after droplet generation (i.e., before swelling) and in the water phase after swelling to equilibrium. Volumes were calculated based on the diameters of 200 microbeads per condition measured by microscopy.

Atomic Force Microscopy Measurements. Glass slides were treated with 3-mercaptopropyl-trimethoxylsilane (MPS; Sigma-Aldrich, U.S.A.) in order to expose reactive thiol groups and covalently attach microgels to the surface of glass slides (Figure S1A). Measurements were carried out at $12 \mu \mathrm{m} / \mathrm{s}$ speed using a NanoWizard II atomic force microscope (JPK Instruments, Germany) and conical silicon nitride probes with a nominal spring constant of $0.65 \mathrm{~N} / \mathrm{m}$
(CSC37, Mikromasch, Switzerland), imposing a Z-length of $6 \mu \mathrm{m}$. Force-distance curves were analyzed with JPK Data Processing software. The Hertz model was applied to calculate the Young's modulus, considering a Poisson's ratio of 0.5 (Figure S1C). Indentation forces were chosen to have a linear relationship between the indentation and the applied force. Four different forces were applied, namely, 80, 100, 120, and $140 \mathrm{nN}$. Young's moduli were calculated as averages of elastic moduli at different indentation forces. Each microbead and bulk gel was analyzed at three different points and measurements were carried out on 10 microbeads and 3 bulk gels.

Bioconjugation of Microgels. Protein functionalization of microgels was carried out by continuous stirring for $2 \mathrm{~h}$ at $37{ }^{\circ} \mathrm{C}$ using protein solutions at a concentration of up to 4-fold molar excess of reactive groups. The $\mathrm{pH}$ of the solution was adjusted to 8 by adding $1 \mathrm{M}$ HEPES buffer solution at $\mathrm{pH}$ 9. Thiolated gelatin and BSA were fluorescently labeled with AlexaFluor594 or AlexaFluor488. In order to functionalize SH-bearing microgels, BSA was PEGylated using maleimide-PEG-NHS. Thiolated gelatin and cysteine-containing RGD were used to functionalize VS- and SH-bearing microcarriers, respectively. Mal-NeutrAvidin, Mal-ProteinA, Alexa488-hIgG, biotinBSA-DsRed, and biotin-FN III ${ }_{9-10}$ were prepared as described. ${ }^{30}$ After protein functionalization, microgels were filtered using $70 \mu \mathrm{m}$ cell strainers and washed several times with PBS to remove unbound proteins. Reduction of disulfide bonds with TCEP (10 mM) was performed on both Alexa488-hIgG and DsRed-BSA-biotin functionalized microcarriers to assess the effect on protein functionalization. A sulfhydryl blocking agent, ethyl iodoacetate $(36 \mathrm{mM})$, was used to quench all thiol groups that did not react with maleimide in order to reduce nonspecific binding (Figure S3A,B). A second washing step was performed at the end of the process to remove unbound proteins. 

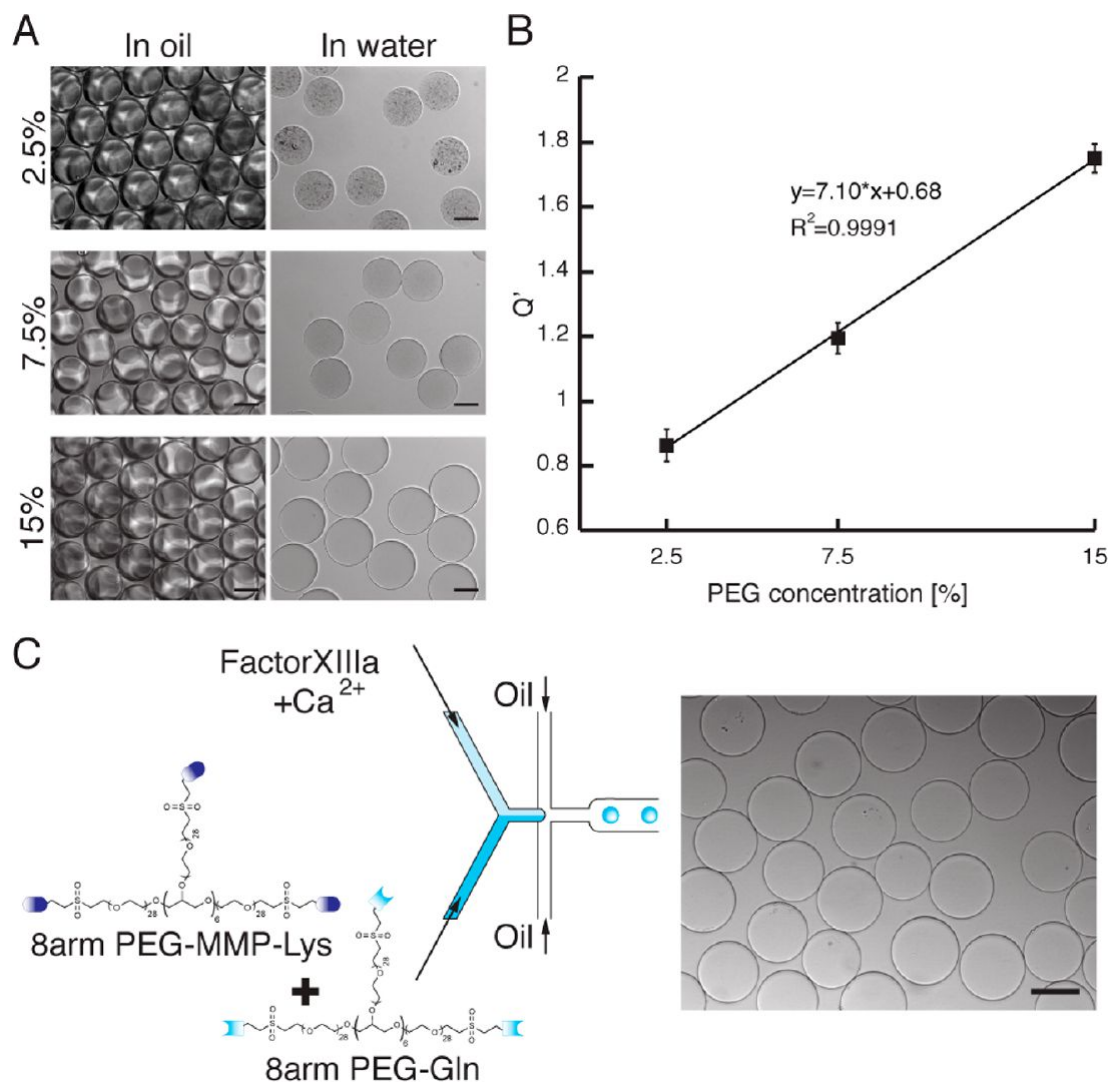

8arm PEG-Gln

Figure 3. Generation of microgels with different cross-linking densities. (A) Micrographs showing microgels generated with a final PEG concentration of $2.5 \%, 7.5 \%$, and $15 \%(\mathrm{w} / \mathrm{v})$ in the oil phase and in water. Images show how microgels swell more at higher PEG concentration. Microgels were generated with a flow rate ratio of 5 and an oil flow rate of $20 \mu \mathrm{L} / \mathrm{min}$. (B) Swelling ratio $\left(Q^{\prime}\right)$ of hydrogel microbeads showing a nearly perfect linear correlation between the PEG concentration and $Q^{\prime}$. (C) Microgels formed from softer PEG-co-peptide hydrogels cross-linked via the transglutaminase factor XIII. The final PEG concentration was $2.32 \%(\mathrm{w} / \mathrm{v})$. Scale bars $=100 \mu \mathrm{m}$.

Image Analysis. Images were acquired with an inverted Olympus IX81 CellR microscope. Fluorescence measurements were performed using a Matlab script on 15 images per condition, with approximately 10 microgels each. Images were first rescaled to a normalized scale based on homogeneous imaging setup, the objects in the foreground were identified and a mask was generated based on bright-field images. A watershed function was used to automatically label beads. Finally, the average intensity per bead was calculated. Statistical $t$ test (type 2, number of tails 2) was performed on the samples; $* * * p<0.001$ and $*_{p}<0.05$.

Adult Stem/Progenitor Cell Culture on Microgels. C2C12 (p7) and placenta-derived human MSCs (isolated and generously provided by M. Ehrbar and P. Lienemann et al., ${ }^{31}$ Department of Obstetrics, University of Zurich Hospital, Switzerland) were cultured on tissue culture flasks in Dulbecco's Modified Eagle Medium (DMEM) with $10 \%$ Fetal Bovine Serum (FBS) or $10 \%$ Fetal Calf Serum (FCS), pen-strep $(10 \mathrm{mg} / \mathrm{mL})$, and sodium pyruvate $(1 \mathrm{mM}$, Gibco). After dissociation with trypsin (TripLE express, Gibco), cells were cultured on microcarriers in a suspension bioreactor (BioLevitator, Hamilton, Switzerland). During the inoculation phase, the rotation speed of the tubes was $50 \mathrm{rpm}$ with an agitation period of 2 min for an entire duration of $4 \mathrm{~h}$. The agitation pause duration was set at $10 \mathrm{~min}$ for $\mathrm{C} 2 \mathrm{C} 12$ cells and MSCs and $10 \mathrm{~min}$ for ESC to minimize microcarrier "bridging". During cell culture, the rotation speed was increased to $80 \mathrm{rpm}$ for all cell types. C2C12 (and ESC, see below) were seeded on microcarriers at a density of $14200 \mathrm{cells} / \mathrm{cm}^{2}$ (corresponding to ca. 10 cells per carrier), whereas the larger MSCs were seeded at a density of 2800 cells $/ \mathrm{cm}^{2}$ (ca. 2 cells per carrier). A total of $6 \mathrm{~mL}$ of medium was used during the inoculation phase (to maximize cell-microgel contact) and $20 \mathrm{~mL}$ during cell culture. Medium was replaced every two days by collecting all the microcarriers on the bottom of the tube by a magnet.
Microgel-based ESC Expansion. R1 Oct4-GFP embryonic stem cells (ESC) were kindly provided by the Zandstra Laboratory (University of Toronto, Canada). ESC were cultured on $0.2 \%$ gelatin-coated Petri dishes in DMEM with $15 \%$ ESC Screened FBS (Fisher), pen-strep $(10 \mathrm{mg} / \mathrm{mL}$, Invitrogen), sodium pyruvate $(1 \mathrm{mM}$, Gibco), nonessential amino acids (0.1 $\mathrm{mM}$, Invitrogen), $\beta$ mercaptoethanol (0.1 mM, Life Technologies), and LIF $\left(10^{3} \mathrm{U} / \mathrm{mL}\right.$, Millipore). ESCs were cultured on PEG microcarriers in bioreactors as described above. Cells were counted every day by retrieving $1 \mathrm{~mL}$ of sample from the spinner and by passing them through a $70 \mu \mathrm{m}$ cell strainer. Microgels were rinsed twice with PBS and trypsinized (TripLE express, Gibco) for $5 \mathrm{~min}$. The resulting solution containing cells and microgels was filtered with a strainer and the cells were counted using a trypan blue exclusion test. Cells were analyzed by flow cytometry using Cyan ADPS analyzer (Beckman Coulter). Data analysis was performed with FlowJo software.

\section{RESULTS AND DISCUSSION}

Characterization of Microfluidic Droplet Generation. To select the most suitable system for the generation of aECM microgels, we first reproduced existing microfluidic droplet formation strategies based on a $\mathrm{T}$-junction ${ }^{32-34}$ and a flow focusing design. ${ }^{35-37}$ Both designs were evaluated at variable geometries and flow rate ratios, which we define as the ratio of flow rates of the aqueous (discontinuous) and oil (continuous) phase (Figure 1). By keeping the total water flow rate constant (here: $4 \mu \mathrm{L} / \mathrm{min} ; 2 \mu \mathrm{L} / \mathrm{min}$ for each inlet based on some of our earlier microfluidic work ${ }^{38}$ ), depending on the flow rate ratio, droplets of spherical or plug-like shape between ca. 90 and 700 $\mu \mathrm{m}$ were formed (Figure S2A). Droplet size decreased with 

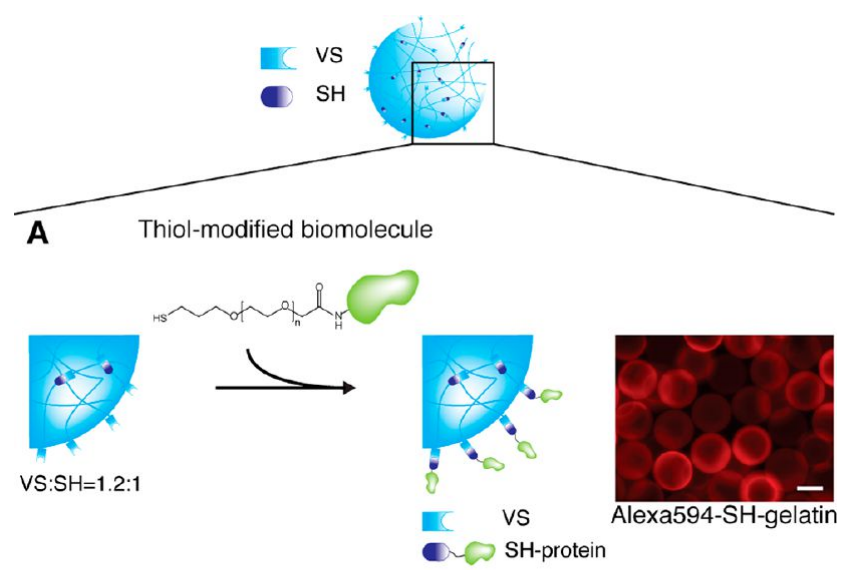

B Maleimide-modified biomolecule

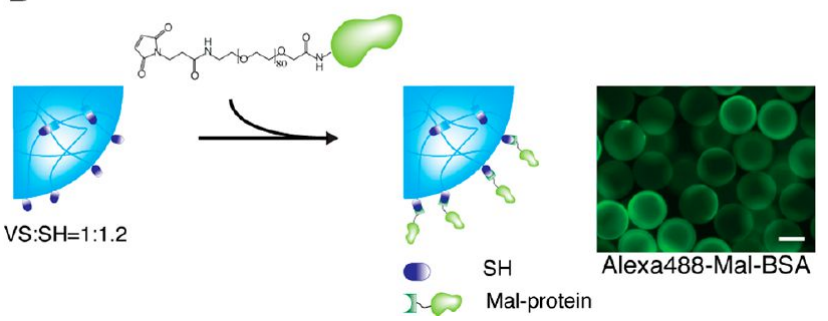

Figure 4. Bioconjugation of PEG microgels. (A) Microgels with molar excess of VS groups (here: 20\%) can be functionalized with thiolated biomolecules (here: fluorescent thiol-activated gelatin). (B) Microgels with molar excess of SH groups (here: $20 \%$ ) can be functionalized with SH-reacting biomolecules (here: fluorescent maleimide-activated BSA). Scale bars $=100 \mu \mathrm{m}$. increasing flow rate ratio (Figure 1B,C), as was expected based on published data. ${ }^{35,36}$ Moreover, at constant flow rate ratio, droplet size increased with microchannel width. Notably, at the same flow rate ratio, droplet dimensions do not change significantly with water flow rate. The stability of the system was better for high flow rate ratios (Figure S2C). Taken together, in our hands, microdroplets with variable dimensions can be readily generated, consistent with earlier work. $35,36,39$

We next calculated the frequency of droplet formation defined as the ratio between the flow rate of the discontinuous phase and the volume of the droplet for a specific flow rate ratio. ${ }^{40}$ We found a frequency range between about $1 \mathrm{~Hz}$ for wide channels at low flow rate ratios and $700 \mathrm{~Hz}$ for narrow channels at high flow rate ratios. However, using smaller channel dimensions and higher flow rate ratios, smaller droplets have been generated at frequencies up to tens of $\mathrm{kHz}^{36}$ Because, in our hands, the flow focusing design allowed for better control of droplet dimensions and stability across a wide size range, as well as higher frequencies of droplets of dimensions in the range of commercially available microcarriers (100-300 $\mu \mathrm{m}$ Figure 1D and Figure S2B), we chose this design to generate aECM microgels.

Generation of Microgels with Well-Defined Size and Physicochemical Properties. PEG-based microgels were generated by forming droplets composed of mixtures of equal volumes of two aqueous precursors containing reactive PEG macromers. Specifically, the mixing of vinyl sulfone (VS)terminated 8-arm-PEG in one channel and thiol (SH)terminated 4-arm-PEG in the other channel resulted in hydrogel formation by stepwise copolymerization via Michael-
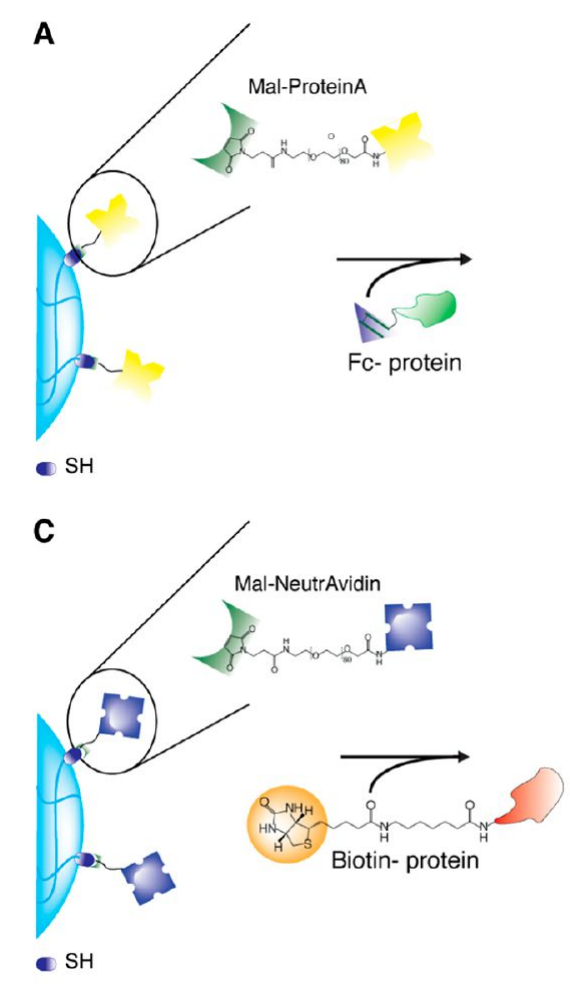
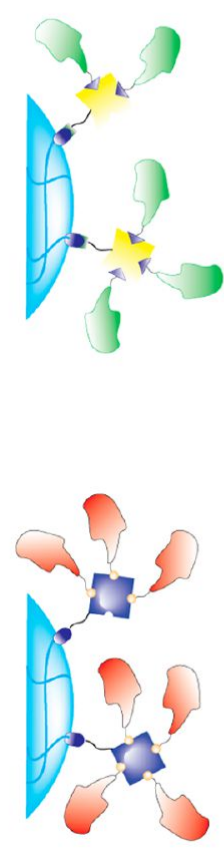

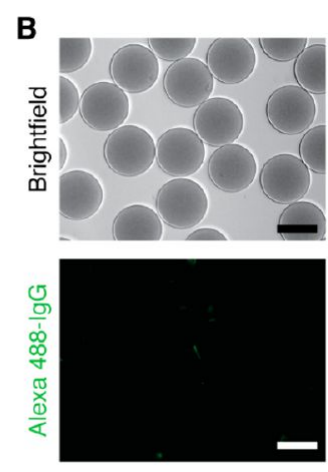

no PrA-lgG

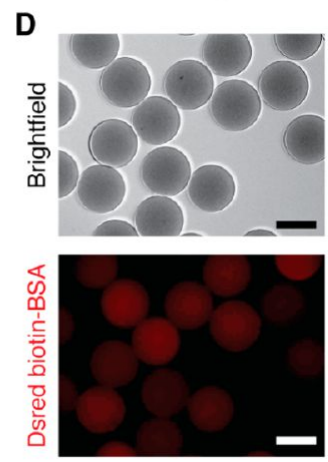

no NA-BSA

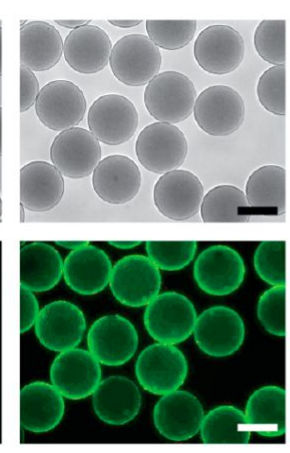

PrA-lgG

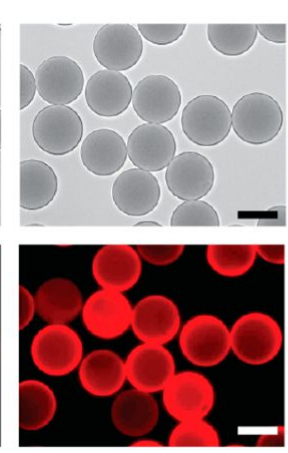

NA-BSA
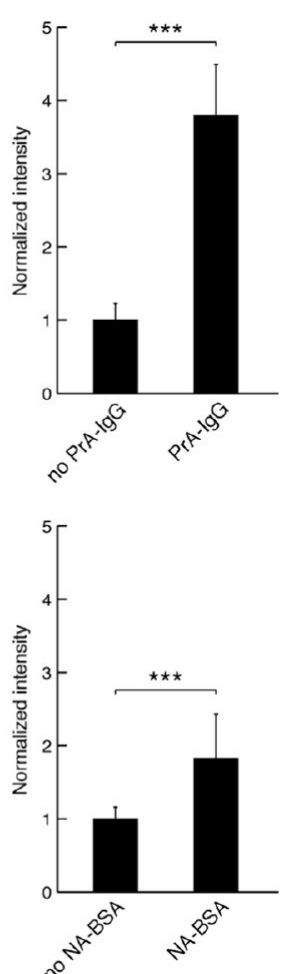

Figure 5. Bioconjugation of microgels via affinitiy binding. (A, C) Microgels with a molar excess of SH groups functionalized with maleimideProteinA or maleimide-NeutrAvidin to capture Fc- or biotin-tagged biomolecules, respectively. (B, D) Examples of microgels functionalized with ProteinA/IgG and NeutrAvidin/biotin-BSA. Measured fluorescence intensities were normalized to the intensity of the negative control (i.e., microcarriers not functionalized with ProteinA or NeutrAvidin). Quantification shows that fluorescent intensities from modified microcarriers are significantly higher. Scale bars $=100 \mu \mathrm{m}$. 
A

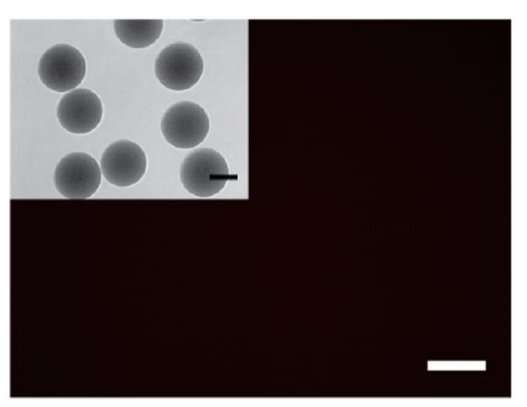

$\lg G: \operatorname{Pr} A=0: 1$

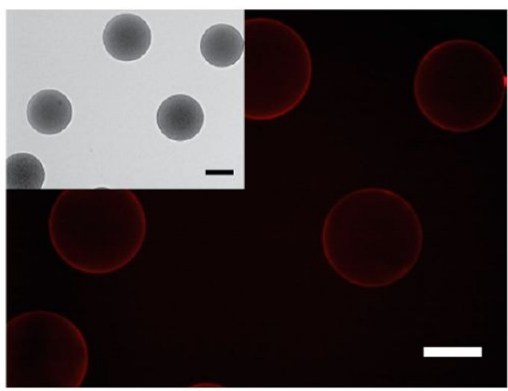

$\lg \mathrm{P}: \operatorname{Pr} A=1: 1$

B

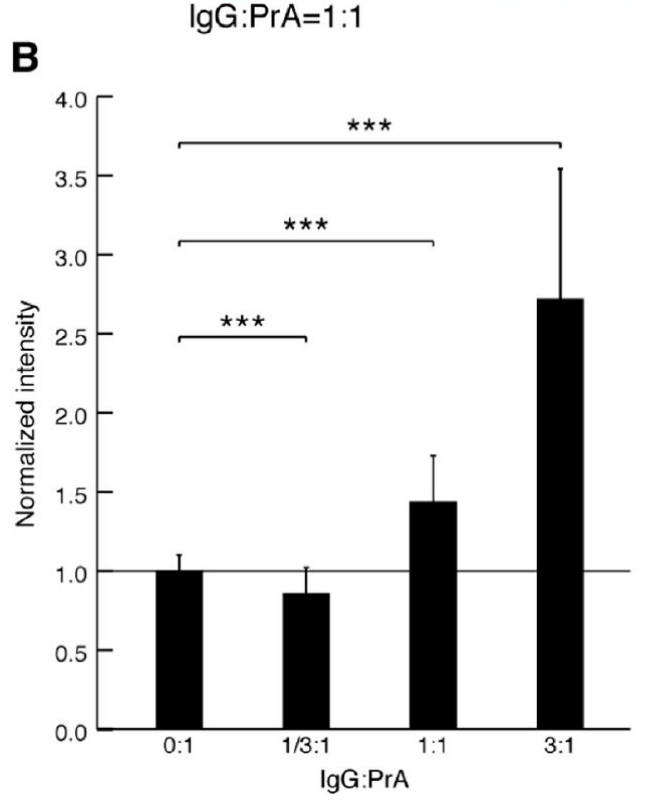

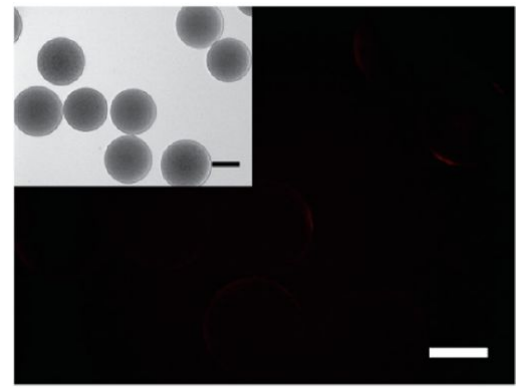

$\lg G: \operatorname{Pr} A=1 / 3: 1$

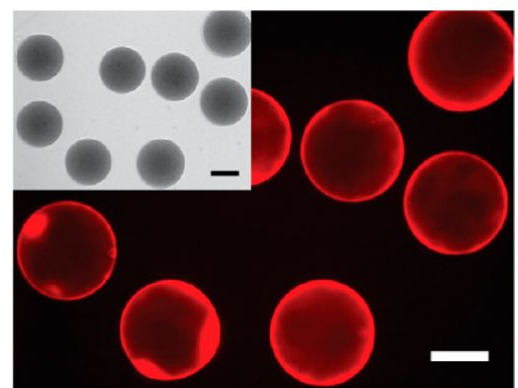

$\lg G: \operatorname{PrA}=3: 1$

Figure 6. Modulation of ligand density on microgel surface. (A) ProteinA-modified microgels functionalized with different amounts of Alexa546 IgG: IgG/PrA = 3:1 (IgG surface density $\left.3.25 \mathrm{nmol} / \mathrm{cm}^{2}\right) ; \operatorname{IgG} / \operatorname{PrA}=1: 1\left(\mathrm{IgG}\right.$ surface density $\left.1.08 \mathrm{nmol} / \mathrm{cm}^{2}\right) ; \mathrm{IgG} / \mathrm{PrA}=1 / 3: 1(\mathrm{IgG}$ surface density $0.36 \mathrm{nmol} / \mathrm{cm}^{2}$ ). (B) Image-based fluorescence analysis. Microgel intensity values were normalized to the control (i.e., no functionalization with $\mathrm{IgG}$ ). Functionalization with increasing amount of $\mathrm{IgG}$ results in significantly higher fluorescent intensities. Scale bars $=100 \mu \mathrm{m}$.

type addition (Figure 2A). ${ }^{28,30}$ Importantly, the laminar flow regime in the microfluidic channels avoids premature mixing and reaction of the two precursors, ensuring a precise control of the volumes mixed in each individual bead (Movie S1). ${ }^{42-44}$ As a result, the size distribution of microgels that was obtained after removal of the oil phase was very narrow (Figure 2B). ${ }^{35,44}$

The chosen channel geometry is particularly well suited for the generation of multicomponent microgels that can, for example, be obtained by loading different gel components into the precursors streams. For instance, in order to facilitate medium exchange during bioreactor cell culture, magnetic particles were loaded into one of the precursor streams to irreversibly trap them in the microgels. Vibrating sample magnetometer analysis demonstrates that the resulting microgels are indeed paramagnetic (Figure 2C).

Importantly, by varying the concentration of the PEG precursors between 2.5 and $15 \%(\mathrm{w} / \mathrm{v})$, we obtain microgels with different cross-linking densities and thus physicochemical properties such as swelling ratios (Figure 3A). Using the above PEG macromere formulations, gel swelling scales are perfectly linear with PEG concentration (Figure 3B).

Atomic force microscopy was used to measure elastic moduli of microgels and bulk gels (Figure S1A). At a concentration of $7.5 \%$, we found that elastic moduli of microgels and bulk gels match well, suggesting that the cross-linking reaction occurs with similar efficiencies at micro- and macro-scale. This may not be surprising as hydrogelation by stepwise copolymeriza- 
A

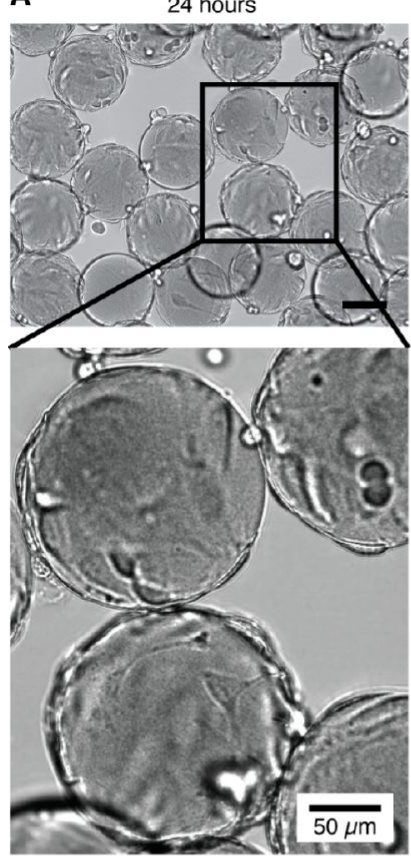

B

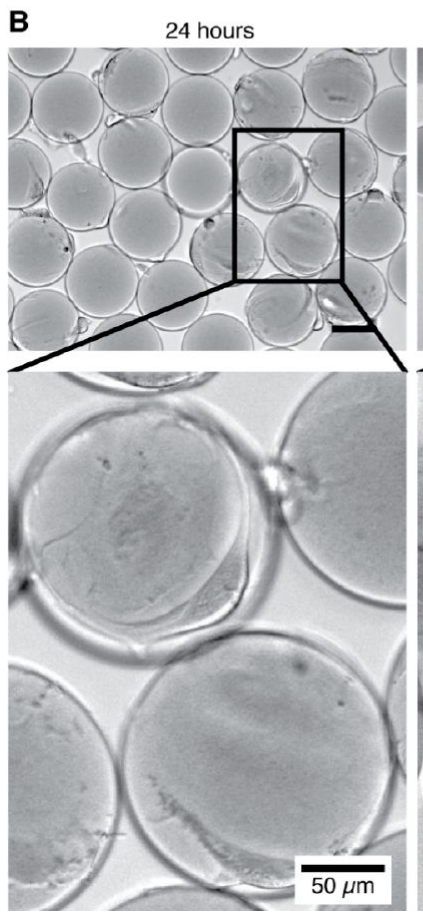

48 hours

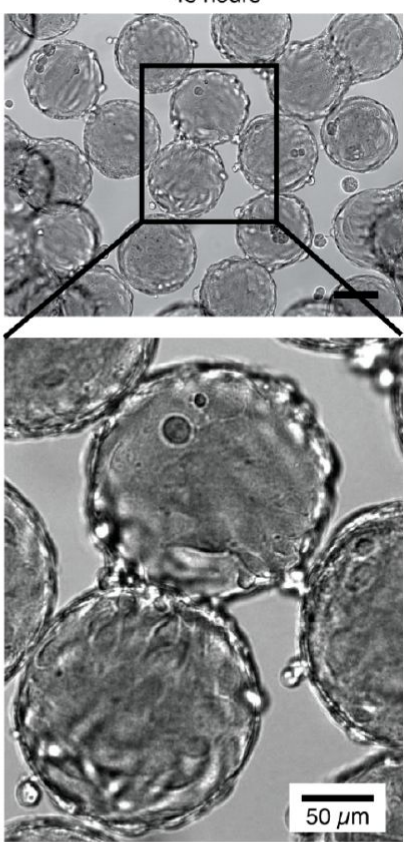

48 hours
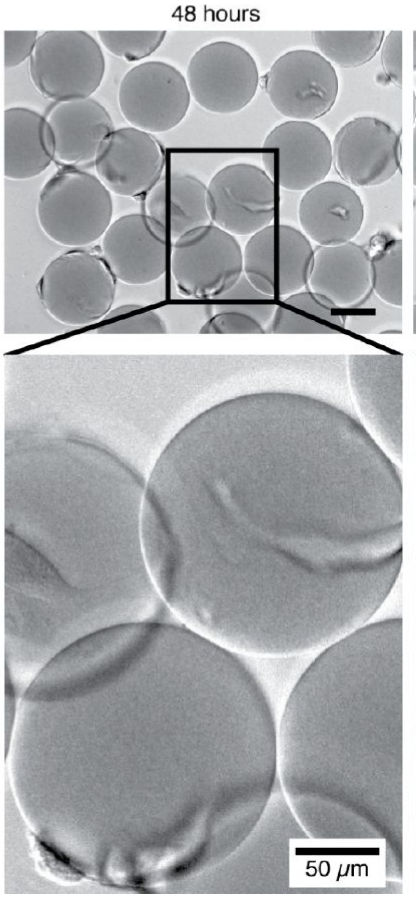
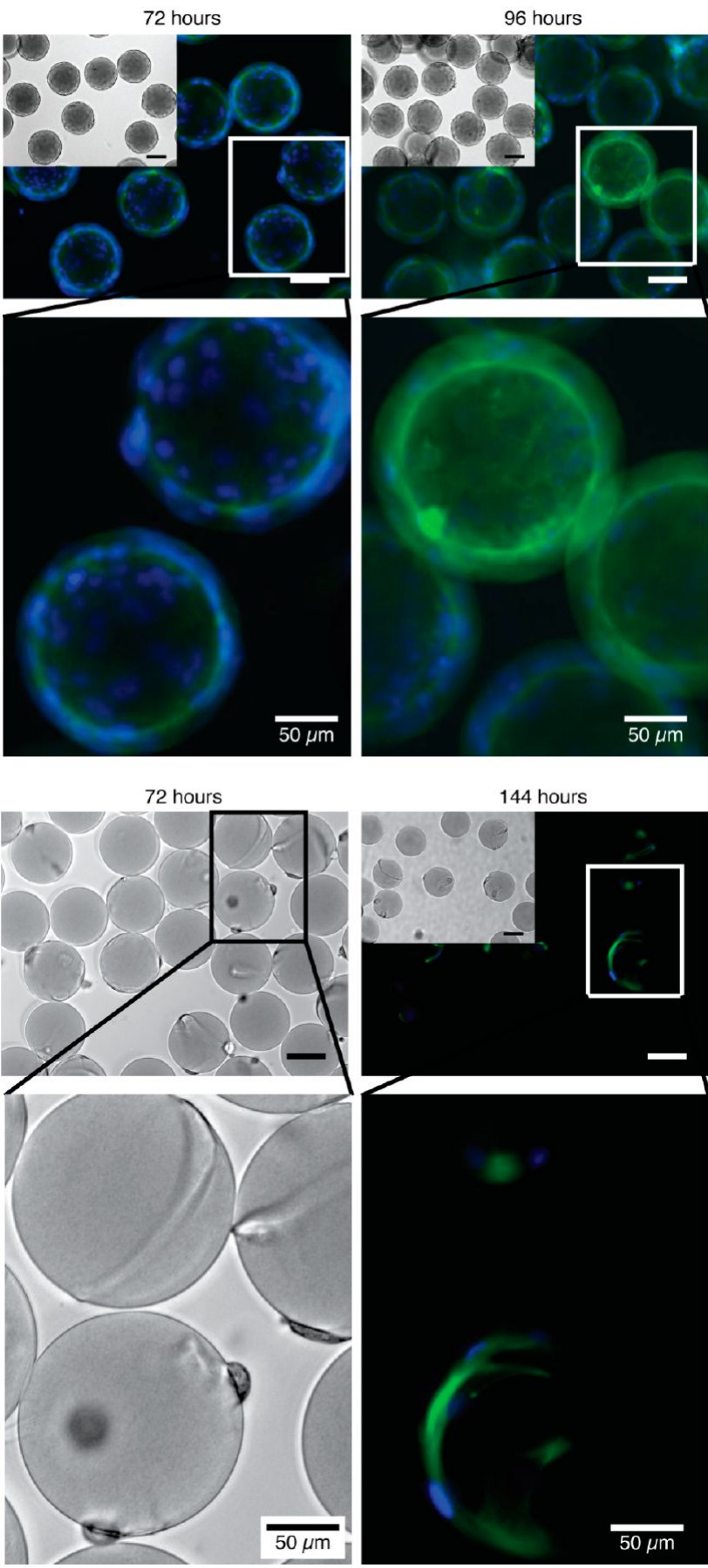

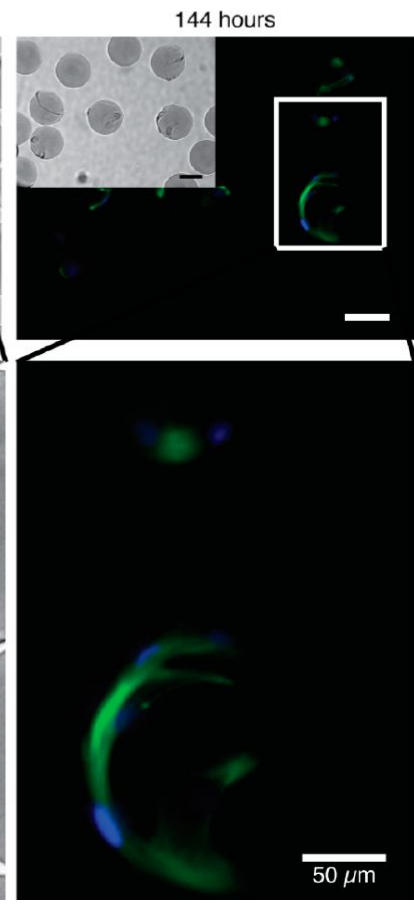

Figure 7. Stem cell adhesion and growth on PEG microgels in bioreactor culture. (A) C2C12 cultured on RGD-functionalized microgels. (B) MSCs cultured on FN III $9-10$-functionalized microgels. Fluorescence micrographs depict phalloidin (cytoskeleton) and DAPI (nuclei) staining for both cell types on the last day of culture. Magnifications indicate cell spreading on microgels at different time points. Scale bars $=100 \mu \mathrm{m}$.

tion via Michael-type addition is an efficient process. ${ }^{28}$ Specifically, at $20 \%$ molar excess of VS groups, we obtain Young's moduli of $28.84 \pm 4.86$ and $31.28 \pm 0.42 \mathrm{kPa}$ for $7.5 \%$ microgels and bulk gels, respectively (Figure S1E). At $20 \%$ molar excess of $\mathrm{SH}$ groups, we obtained elastic moduli of 28.1 \pm 5.02 and $32.99 \pm 6.21 \mathrm{kPa}$ for microgels and bulk gels, respectively.

Notably, microgels can also be formed from much softer PEG-co-peptide hydrogels formulations such as those crosslinked via Michael-type addition ${ }^{28}$ (not shown) or the transglutaminase factor XIII (FXIIIa) ${ }^{45}$ (Figure 3C). For instance, at a PEG concentration of $2.3 \%(\mathrm{w} / \mathrm{v})$, FXIIIacross-linked microbeads with a modulus of about $750 \mathrm{~Pa}$ were obtained. Therefore, droplet microfluidics is suitable to generate PEG-based microgels across a very wide range of cross-linking densities and mechanical properties (e.g., from hundreds of $\mathrm{Pa}$ up to tens of $\mathrm{kPa}$ in Young's modulus).

Bioconjugation of PEG Microgels via Covalent Coupling. Next, a bioconjugation protocol was developed to modify the above, relatively inert microgels with biomolecules of interest (Figure 4). To this end, microgels were formed at a slightly imbalanced stoichiometric ratio of functional groups 
A

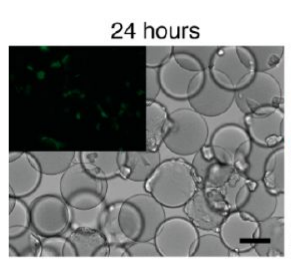

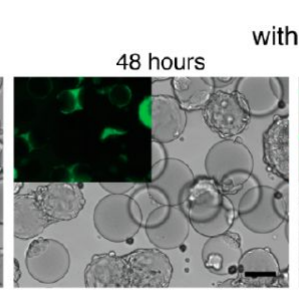

with LIF

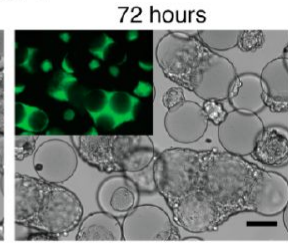

without LIF
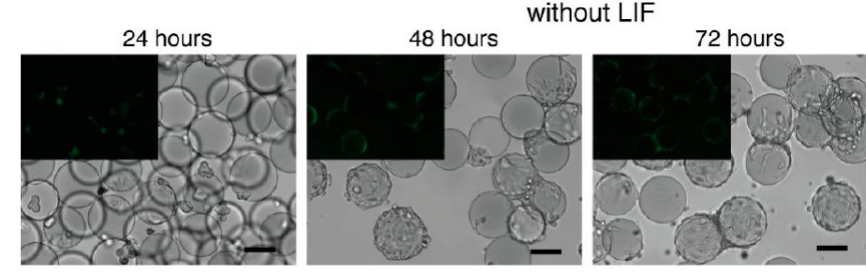
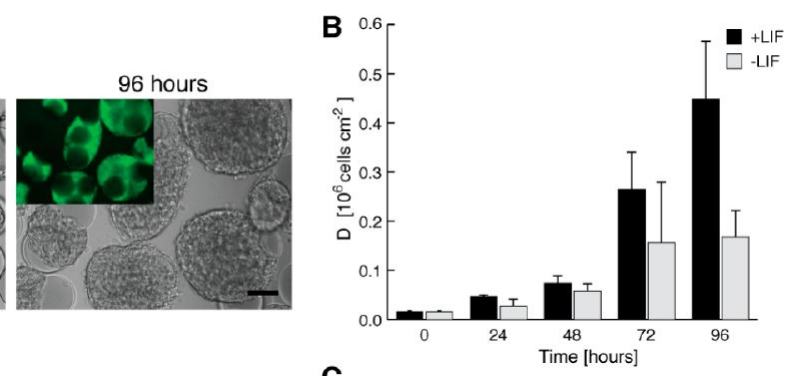

C

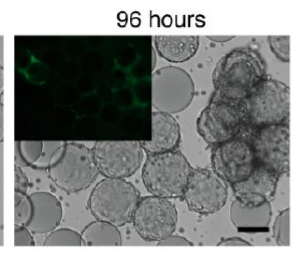

Figure 8. ESC expansion on PEG microgels in bioreactor cultures. (A) ESC cultured on gelatin-functionalized microgels in the presence or absence of LIF. Fluorescence micrographs in the left corners indicate Oct4 expression. (B) ESC growth curves depicting cell density $(D)$ for different culture periods. In presence of LIF, cells proliferate more extensively. Partial cell detachment can be observed after 4 days of culture in the absence of LIF. (C) GFP expression measured by flow cytometry. In the presence of LIF, ESCs maintain high Oct4 expression up to day 4. In the absence of LIF, Oct4 expression drops dramatically, suggesting ESC differentiation. Scale bar $=100 \mu \mathrm{m}$.

such that free reactive groups on bead surfaces could be used as "chemical handles" to tether bioactive ligands. PEG precursor streams of different final concentrations were thus mixed to achieve an up to $20 \%$ molar excess of one of the reactive moieties (VS or $\mathrm{SH}$ ) producing such “activated" gel surfaces. Networks bearing free VS groups can then be functionalized via Michael-type addition with thiolated biomolecules (Figure 4A), whereas SH-presenting networks are amenable for functionalization with biomolecules bearing unsaturated groups such as vinylsulfones, maleimides, acrylates, or acrylamides. Due to its excellent specificity toward reaction with thiols, we chose maleimide groups to demonstrate the latter bioconjugation strategy (Figure 4B).

An estimation of the maximal protein amount that can be tethered to the microcarrier surface was carried out, taking into account the dry mass of the gel and the molar excess of the reactive VS or $\mathrm{SH}$ groups. Assuming a layer (or shell) of $10 \mathrm{~nm}$ on the surface of microcarriers that is accessible for cells to interact with, we obtain a protein surface density of about 3.45 $\mathrm{nmol} / \mathrm{cm}^{2}$ for $7.5 \%(\mathrm{w} / \mathrm{v})$ microcarriers bearing a $20 \%$ molar excess of VS groups. Similarly, at a $20 \%$ molar excess of $\mathrm{SH}$ groups, $7.5 \%$ microcarriers yield a maximum of about 3.25 $\mathrm{nmol} / \mathrm{cm}^{2}$ of protein.

Bioconjugation of PEG Microgels Using Affinity Binding Schemes. The versatility of the chosen two-step bioconjugation strategy allows for functionalization of PEG microgels using other strategies such as affinity-based protein capture. $^{30,38}$ This strategy could be particularly interesting for modifying microcarriers with recombinant proteins such as growth factors that are often obtained as "chimeric" proteins containing a tag for purification (e.g., Fc-tag or His-tag). For example, ProteinA or NeutrAvidin can be tethered to thiolbearing microgels using a heterofunctional maleimide-PEGNHS linker (Figure 5A,C). These microgels efficiently capture Fc-tagged or biotinylated proteins as shown in Figure 5. Indeed, fluorescent intensity measurements and image analysis revealed specific binding of fluorescent model proteins (IgG and biotinylated BSA) on ProteinA- or NeutrAvidin-modified microgels (Figure 5B,D). Of note, a reduction of disulfide bonds by tris(2-carboxyethyl)phosphine hydrochloride does not increase the efficiency of functionalization (Figure S3).

Next, to demonstrate the possibility to modulate the surface density of biochemical factors, we functionalized ProteinAmodified microgels with different molar excess of fluorescent IgG (Figure 6A). Quantification of fluorescent intensities by image analysis reveals a significant increase in concentration with increasing molar excess of IgG (Figure 6B). Therefore, our bioconjugation strategy can be applied to control the concentration of tethered biomolecules.

Peptide or Protein-Functionalized PEG Microgels Promote Stem/Progenitor Cell Adhesion and Growth. To validate our aECM microgels in bioreactor-based cell culture, selective peptide- and protein-modified formulations were tested for their ability to promote stem/progenitor cell adhesion and proliferation (Figure 7 ). To this end, VS-bearing microcarriers were functionalized with cysteine-containing RGD peptide and used to expand $\mathrm{C} 2 \mathrm{C} 12$ muscle progenitor cells (Figure 7A). Moreover, microcarriers presenting an excess of thiols were functionalized with maleimide-activated NeutrAvidin and then with biotinylated fibronectin fragment FN $\mathrm{III}_{9-10}$ for human mesenchymal stem cell (MSC) culture (Figure 7B). Micrographs show very good spreading and adhesion of both cell types on these bioactive PEG microgels. Compared to $\mathrm{C} 2 \mathrm{C} 12$ cultures, a relatively low number of MSCs was observed on microgels which we attribute to the low initial cell seeding density (ca. 2800 cells $/ \mathrm{cm}^{2}$ ) and their slower growth rate, as very few cells were found to detach from the microgels (Figure 7B).

Gelatin-Functionalized PEG Microgels Promote Mouse ESC Expansion. As a tractable model system to probe stem cell expansion on our aECM microgels, we used an ESC reporter line wherein GFP marks cells that express the pluripotency transcription factor Oct4. To promote efficient ESC adhesion, PEG microgels bearing excess VS groups were modified with thiolated gelatin, a protein that is routinely used in ESC expansion culture on plastic dishes. 
In the presence of leukemia inhibitory factor (LIF), aECM microgels promote expansion of Oct4-positive cells under bioreactor culture conditions (Figure 8A). Cells tended to form large clusters that often spanned over multiple carriers, a phenomenon that was previously reported. ${ }^{16,17,46}$ Upon LIF removal, cells rapidly lost GFP expression, formed monolayers instead of larger clusters and finally detached from the carriers upon reaching confluency. Moreover, further assessment of ESC fate by flow cytometry revealed significantly less proliferation (Figure 8B) and lower numbers of GFP-positive cells (Figure $8 \mathrm{C}$ ) in the absence of LIF. Of note, these experiments also show that cells can be readily harvested from the microgels and used for further analyses such as flow cytometry or manipulation of cell fate in other culture formats.

\section{CONCLUSIONS}

Here we demonstrate the application of droplet microfluidics for the reliable generation of monodisperse microgels with extremely well defined biochemical and biophysical properties. The versatility of the chosen approach allows us to combine multiple gel components and additives (such as magnetic particles) almost at will, providing the exciting opportunity to generate a huge diversity of microgels for cell manipulation and screening applications, for example to discover hydrogel formulations that allow the manipulation of difficult-to-culture cell types. Our droplet-based aECM fabrication scheme should also be useful to encapsulate cells in $3 \mathrm{D} .^{26,44,47-49}$

\section{ASSOCIATED CONTENT}

\section{S Supporting Information}

Additional data are provided. Figure S1 provides further information about AFM on microgels and bulk gels formed at different VS/SH molar ratios. Figure S2 reports the droplet frequency generation for different microchannel layouts. Figure S3 shows fluorescent pictures and measurements carried out on microcarriers functionalized via affinity binding scheme and treated with TCEP and ethyl iodoacetate. This material is available free of charge via the Internet at http://pubs.acs.org.

\section{AUTHOR INFORMATION}

\section{Corresponding Author}

*E-mail: matthias.lutolf@epfl.ch.

\section{Present Address}

${ }^{\dagger}$ Biomechanics Orthopedics Laboratory, Institute of Bioengineering, Ecole Polytechnique Fédérale de Lausanne (EPFL).

\section{Notes}

The authors declare no competing financial interest.

\section{ACKNOWLEDGMENTS}

This work was supported by a European Young Investigator (EURYI) Award (PE002-117115/1) to M.P.L. We thank Adrien de Tonnac, Nanna Torpe-Jorgensen, Steffen Cosson, and Stefan Kobel for contributing to the development of droplet microfluidics in our lab, Andrea Negro for support with image analysis, Domenica Convertino and members of the Laboratory of Cell Biophysics at EPFL for precious help with AFM measurements, and Samy Gobaa and Marta Roccio for valuable technical advice. We also thank Ann Marie Hirt who helped with the vibrating sample magnetometer at LNM at ETH Zurich.

\section{REFERENCES}

(1) Seliktar, D. Designing Cell-Compatible Hydrogels for Biomedical Applications. Science 2012, 336 (6085), 1124-1128.

(2) DeForest, C. A.; Anseth, K. S. Advances in Bioactive Hydrogels to Probe and Direct Cell Fate. Annual Review of Chemical and Biomolecular Engineering; Annual Reviews: Palo Alto, CA, 2012, Vol 3, 3, pp 421-444.

(3) Lutolf, M. P.; Hubbell, J. A. Synthetic Biomaterials As Instructive Extracellular Microenvironments for Morphogenesis in Tissue Engineering. Nat. Biotechnol. 2005, 23 (1), 47-55.

(4) Lutolf, M. P.; Blau, H. M. Artificial Stem Cell Niches. Adv. Mater. 2009, 21 (32-33), 3255-3268.

(5) Wagers, A. J. The Stem Cell Niche in Regenerative Medicine. Cell Stem Cell 2012, 10 (4), 362-369.

(6) Gauvin, R.; Parenteau-Bareil, R.; Dokmeci, M. R.; Merryman, W. D.; Khademhosseini, A. Hydrogels and Microtechnologies for Engineering the Cellular Microenvironment. Nanomed. Nanobiotechnol. 2012, 4 (3), 235-246.

(7) Du, Y. A.; Lo, E.; Ali, S.; Khademhosseini, A. Directed Assembly of Cell-Laden Microgels for Fabrication of 3D Tissue Constructs. Proc. Natl. Acad. Sci. U.S.A. 2008, 105 (28), 9522-9527.

(8) Khademhosseini, A.; Langer, R.; Borenstein, J.; Vacanti, J. P. Microscale Technologies for Tissue Engineering and Biology. Proc. Natl. Acad. Sci. U.S.A. 2006, 103 (8), 2480-2487.

(9) Li, C. Y.; Wood, D. K.; Hsu, C. M.; Bhatia, S. N. DNATemplated Assembly of Droplet-Derived PEG Microtissues. Lab Chip 2011, 11 (17), 2967-2975.

(10) Matsunaga, Y. T.; Morimoto, Y.; Takeuchi, S. Molding Cell Beads for Rapid Construction of Macroscopic 3D Tissue Architecture. Adv. Mater. 2011, 23 (12), H90-H94.

(11) Chen, A. A.; Underhill, G. H.; Bhatia, S. N. Multiplexed, HighThroughput Analysis of 3D Microtissue Suspensions. Integr. Biol. 2010, 2 (10), 517-527.

(12) King, J. A.; Miller, W. M. Bioreactor Development for Stem Cell Expansion and Controlled Differentiation. Curr. Opin. Chem. Biol. 2007, 11 (4), 394-398.

(13) Serra, M.; Correia, C.; Brito, C.; Sousa, M. F. Q.; Rostoes, R. M.; Jensen, J.; Bjorquist, P.; Carrondo, M. J. T.; Alves, P. M. Process Engineering of Human Embryonic Stem Cells for Clinical Application. Hum. Gene Ther. 2011, 22 (10), A56-A56.

(14) Vanwezel, A. L. Growth of Cell-Strains and Primary Cells on Micro-Carriers in Homogeneous Culture. Nature 1967, 216 (5110), 64-\&.

(15) Reuveny, S., Microcarrier Culture Systems. In Large-Scale Mammalian Cell Culture Technology; Lubiniecki, A. S., Ed.; Marcel Dekker, Inc.: New York, 1990; pp 271-341.

(16) Fok, E. Y. L.; Zandstra, P. W. Shear-Controlled Single-Step Mouse Embryonic Stem Cell Expansion and Embryoid Body-Based Differentiation. Stem Cells 2005, 23 (9), 1333-1342.

(17) Abranches, E.; Bekman, E.; Henrique, D.; Cabral, J. M. S. Expansion of Mouse Embryonic Stem Cells on Microcarriers. Biotechnol. Bioeng. 2007, 96 (6), 1211-1221.

(18) Fernandes, A. M.; Fernandes, T. G.; Diogo, M. M.; da Silva, C. L.; Henrique, D.; Cabral, J. M. S. Mouse Embryonic Stem Cell Expansion in a Microcarrier-Based Stirred Culture System. J. Biotechnol. 2007, 132 (2), 227-236.

(19) Yang, Y.; Rossi, F. M. V.; Putnins, E. E. Ex Vivo Expansion of Rat Bone Marrow Mesenchymal Stromal Cells on Microcarrier Beads in Spin Culture. Biomaterials 2007, 28 (20), 3110-3120.

(20) Oh, S. K. W.; Chen, A. K.; Mok, Y.; Chen, X. L.; Lim, U. M.; Chin, A.; Choo, A. B. H.; Reuveny, S. Long-term microcarrier suspension cultures of human embryonic stem cells. Stem Cell Res. 2009, 2 (3), 219-230.

(21) Serra, M.; Brito, C.; Leite, S. B.; Gorjup, E.; von Briesen, H.; Carrondo, M. J. T.; Alves, P. M. Stirred Bioreactors for the Expansion of Adult Pancreatic Stem Cells. Ann. Anat. 2009, 191 (1), 104-115.

(22) Eibes, G.; dos Santos, F.; Andrade, P. Z.; Boura, J. S.; Abecasis, M. M. A.; da Silva, C. L.; Cabral, J. M. S. Maximizing the Ex Vivo 
Expansion of Human Mesenchymal Stem Cells Using a MicrocarrierBased Stirred Culture System. J. Biotechnol. 2010, 146 (4), 194-197.

(23) Chen, A. K. L.; Chen, X. L.; Choo, A. B. H.; Reuveny, S.; Oh, S. K. W. Critical Microcarrier Properties Affecting the Expansion of Undifferentiated Human Embryonic Stem Cells. Stem Cell Res. 2011, 7 (2), 97-111.

(24) Zweigerdt, R.; Olmer, R.; Singh, H.; Haverich, A.; Martin, U. Scalable Expansion of Human Pluripotent Stem Cells in Suspension Culture. Nat. Protoc. 2011, 6 (5), 689-700.

(25) Theberge, A. B.; Courtois, F.; Schaerli, Y.; Fischlechner, M.; Abell, C.; Hollfelder, F.; Huck, W. T. S. Microdroplets in Microfluidics: An Evolving Platform for Discoveries in Chemistry and Biology. Angew. Chem., Int. Ed. 2010, 49 (34), 5846-5868.

(26) Velasco, D.; Tumarkin, E.; Kumacheva, E. Microfluidic Encapsulation of Cells in Polymer Microgels. Small 2012, 8 (11), $1633-1642$.

(27) Seemann, R.; Brinkmann, M.; Pfohl, T.; Herminghaus, S. Droplet Based Microfluidics. Rep. Prog. Phys. 2012, 75 (1), 016601.

(28) Lutolf, M. P.; Hubbell, J. A. Synthesis and Physicochemical Characterization of End-Linked Poly(ethylene glycol)-co-peptide Hydrogels Formed by Michael-Type Addition. Biomacromolecules 2003, 4 (3), 713-722.

(29) Martino, M. M.; Mochizuki, M.; Rothenfluh, D. A.; Rempel, S. A.; Hubbell, J. A.; Barker, T. H. Controlling Integrin Specificity and Stem Cell Differentiation in 2D and 3D Environments through Regulation of Fibronectin Domain Stability. Biomaterials 2009, 30 (6), 1089-1097.

(30) Cosson, S.; Kobel, S. A.; Lutolf, M. P. Capturing Complex Protein Gradients on Biomimetic Hydrogels for Cell-Based Assays. Adv. Funct. Mater. 2009, 19 (21), 3411-3419.

(31) Semenov, O. V.; Koestenbauer, S.; Riegel, M.; Zech, N.; Zimmermann, R.; Zisch, A. H.; Malek, A. Multipotent Mesenchymal Stem Cells from Human Placenta: Critical Parameters for Isolation and Maintenance of Stemness after Isolation. Am. J. Obstet. Gynecol. 2010, 202 (2), 193.e1.

(32) Thorsen, T.; Roberts, R. W.; Arnold, F. H.; Quake, S. R. Dynamic Pattern Formation in a Vesicle-Generating Microfluidic Device. Phys. Rev. Lett. 2001, 86 (18), 4163-4166.

(33) Nisisako, T.; Torii, T.; Higuchi, T. Droplet Formation in a Microchannel Network. Lab Chip 2002, 2 (1), 24-26.

(34) Xu, J. H.; Luo, G. S.; Li, S. W.; Chen, G. G. Shear Force Induced Monodisperse Droplet Formation in a Microfluidic Device by Controlling Wetting Properties. Lab Chip 2006, 6 (1), 131-136.

(35) Tan, Y. C.; Cristini, V.; Lee, A. P. Monodispersed Microfluidic Droplet Generation by Shear Focusing Microfluidic Device. Sens. Actuators B 2006, 114 (1), 350-356.

(36) Yobas, L.; Martens, S.; Ong, W. L.; Ranganathan, N. HighPerformance Flow-Focusing Geometry for Spontaneous Generation of Monodispersed Droplets. Lab Chip 2006, 6 (8), 1073-1079.

(37) Seo, M.; Paquet, C.; Nie, Z. H.; Xu, S. Q.; Kumacheva, E. Microfluidic Consecutive Flow-Focusing Droplet Generators. Soft Matter 2007, 3 (8), 986-992.

(38) Allazetta, S.; Cosson, S.; Lutolf, M. P. Programmable Microfluidic Patterning of Protein Gradients on Hydrogels. Chem. Commun. 2011, 47 (1), 191-193.

(39) Li, W.; Young, E. W. K.; Seo, M.; Nie, Z.; Garstecki, P.; Simmons, C. A.; Kumacheva, E. Simultaneous Generation of Droplets with Different Dimensions in Parallel Integrated Microfluidic Droplet Generators. Soft Matter 2008, 4 (2), 258-262.

(40) Trivedi, V.; Ereifej, E. S.; Doshi, A.; Sehgal, P.; VandeVord, P. J.; Basu, A. S. Microfluidic Encapsulation of Cells in Alginate Capsules for High Throughput Screening. EMBC: 2009 Annual International Conference of the IEEE Engineering in Medicine and Biology Society, Minneapolis, MN, Sept 3-6, 2009, IEEE: United States, 2009, Vols 1-20, pp 7037-7040.

(41) Teh, S. Y.; Lin, R.; Hung, L. H.; Lee, A. P. Droplet Microfluidics. Lab Chip 2008, 8 (2), 198-220.

(42) Kim, J. W.; Utada, A. S.; Fernandez-Nieves, A.; Hu, Z. B.; Weitz,

D. A. Fabrication of Monodisperse Gel Shells and Functional
Microgels in Microfluidic Devices. Angew. Chem., Int. Ed. 2007, 46 (11), 1819-1822.

(43) Duncanson, W. J.; Lin, T.; Abate, A. R.; Seiffert, S.; Shah, R. K.; Weitz, D. A. Microfluidic Synthesis of Advanced Microparticles for Encapsulation and Controlled Release. Lab Chip 2012, 12 (12), $2135-2145$

(44) Rossow, T.; Heyman, J. A.; Ehrlicher, A. J.; Langhoff, A.; Weitz, D. A.; Haag, R.; Seiffert, S. Controlled Synthesis of Cell-Laden Microgels by Radical-Free Gelation in Droplet Microfluidics. J. Am. Chem. Soc. 2012, 134 (10), 4983-4989.

(45) Ehrbar, M.; Rizzi, S. C.; Schoenmakers, R. G.; San Miguel, B.; Hubbell, J. A.; Weber, F. E.; Lutolf, M. P. Biomolecular Hydrogels Formed and Degraded via Site-Specific Enzymatic Reactions. Biomacromolecules 2007, 8 (10), 3000-3007.

(46) Alfred, R.; Radford, J.; Fan, J.; Boon, K.; Krawetz, R.; Rancourt, D.; Kallos, M. S. Efficient Suspension Bioreactor Expansion of Murine Embryonic Stem Cells on Microcarriers in Serum-Free Medium. Biotechnol. Prog. 2011, 27 (3), 811-823.

(47) Kumachev, A.; Greener, J.; Tumarkin, E.; Eiser, E.; Zandstra, P. W.; Kumacheva, E. High-Throughput Generation of Hydrogel Microbeads with Varying Elasticity for Cell Encapsulation. Biomaterials 2011, 32 (6), 1477-1483.

(48) Tumarkin, E.; Tzadu, L.; Csaszar, E.; Seo, M.; Zhang, H.; Lee, A.; Peerani, R.; Purpura, K.; Zandstra, P. W.; Kumacheva, E. HighThroughput Combinatorial Cell Co-Culture Using Microfluidics. Integr. Biol. 2011, 3 (6), 653-662.

(49) Tan, W. H.; Takeuchi, S. Monodisperse Alginate Hydrogel Microbeads for Cell Encapsulation. Adv. Mater. 2007, 19 (18), 2696. 